\title{
Advances in Particle Detectors
}

\author{
Junji Haba ${ }^{1}$ \\ High Energy Accelerator Research Organization, KEK \\ Oho 1-1, Tsukuba, Ibaraki, Japan \\ E-mail: junji.haba@kek.jp
}

Particle detectors are essential players of particle physics experiments to explore new regimes of Nature. A detector system in every modern particle experiment consists of hundreds types of advanced elements which detect the particles emitted from the interactions or decay of particles to reconstruct their trajectories in space and time. The most competitive experiments are always equipped with the most advanced detector system to beat others. In this review, some of the recent advances achieved in particle detectors are reviewed.

XXIX International Symposium on Lepton Photon Interactions at High Energies - LeptonPhoton2019 August 5-10, 2019

Toronto, Canada

\section{${ }^{1}$ Speaker}




\section{Introduction}

A detector system in every modern particle experiment consists of hundreds of types of advanced elements which detect the particles emitted from the interactions or decay of particles to reconstruct their trajectories in space and time. The most competitive experiments are always equipped with the most advanced detector system to beat others.

Modern high luminosity colliders like LHC/HL-LHC or SuperKEKB would impose higher and higher fluxes of particles and radiation on the detectors working there. Table 1 summarizes radiation environments assumed in typical collider experiments. [1]

Table 1 Expected particle flux, neutron fluence and total ionization dose in typical collider experiments.

\begin{tabular}{|l|c|c|c|c|c|c|}
\hline & STAR & Belle II & $\begin{array}{c}\text { ALICE } \\
\text { HI }\end{array}$ & ILC & $\begin{array}{c}\text { LHC } \\
\mathrm{pp}\end{array}$ & $\begin{array}{c}\text { HL-LHC } \\
\text { Inner }\end{array}$ \\
\hline $\begin{array}{l}\text { Particle rate } \\
\left(\mathrm{kHz} / \mathrm{mm}^{2}\right)\end{array}$ & 4 & 400 & 10 & 250 & 1,000 & $>10,000$ \\
\hline$\Phi\left(\mathrm{neq} / \mathrm{cm}^{2}\right)$ & $>10^{12}$ & $10^{14}$ & $>10^{13}$ & $10^{12}$ & $2 \times 10^{15}$ & $2 \times 10^{16}$ \\
\hline TID $(\mathrm{Mrad})$ & 0.2 & 20 & 0.7 & 0.4 & 80 & $>1000$ \\
\hline
\end{tabular}

Under such harsh conditions, the detector system should be functional to extract the necessary information of the true traces from the overwhelming background activity. That can be better performed by resolving the signals with higher and higher precision in time as well as in space. In this respect, a history of R\&D in a modern particle detector can be considered as a progressive effort to make the scale of the basic unit of detector smaller to keep its occupancy reasonably low, or in other words, to further improve the precision or resolution of space or time measurements. Stable functionality under severe radiation is another very serious requirement for a detector.

In this short review the most recent result of such improvements of precision in measurement of time or space coordinates and efforts against radiation are briefly summarized.

\section{Improvements of precision in measurement}

\section{Time measurement}

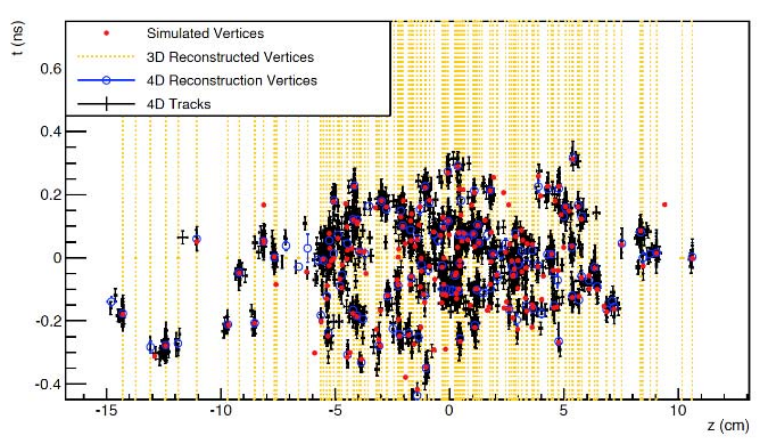

Figure 1 Reconstructed vertices in z-location and time simulated for HL LHC [2]
Timing information with precision better than 1 nanosecond is crucial for detectors in modern high luminosity collider experiments. In the case of HL LHC experiments, 200 pile-up interactions are expected in each bunch crossing. As shown in Fig. 1, spatial information alone is insufficient to provide a good separation among interactions and precise 
reconstruction of interaction timing better than 100 picosecond should be vital to further distinguish the pile-up interactions.

There are lots of R\&D works to implement precise timing measurement of tracks in the LHC detectors using a silicon sensor with internal avalanche gain (LGAD, Low Gain Avalanche Diode) [3] or a segmented fast scintillator. The latter case are intensively studied by the CMS R\&D teams as MTD (Mip Timing Detector). Their recent study [4] demonstrates that timing resolution of 30 picosecond can be otained for the LYSO (Cerium doped Lutetium Yttrium Orthosilicate) scintillator rod of $3 \times 3 \times 50 \mathrm{~mm}^{3}$, as shown in Fig. 2 The basic design of the MTD
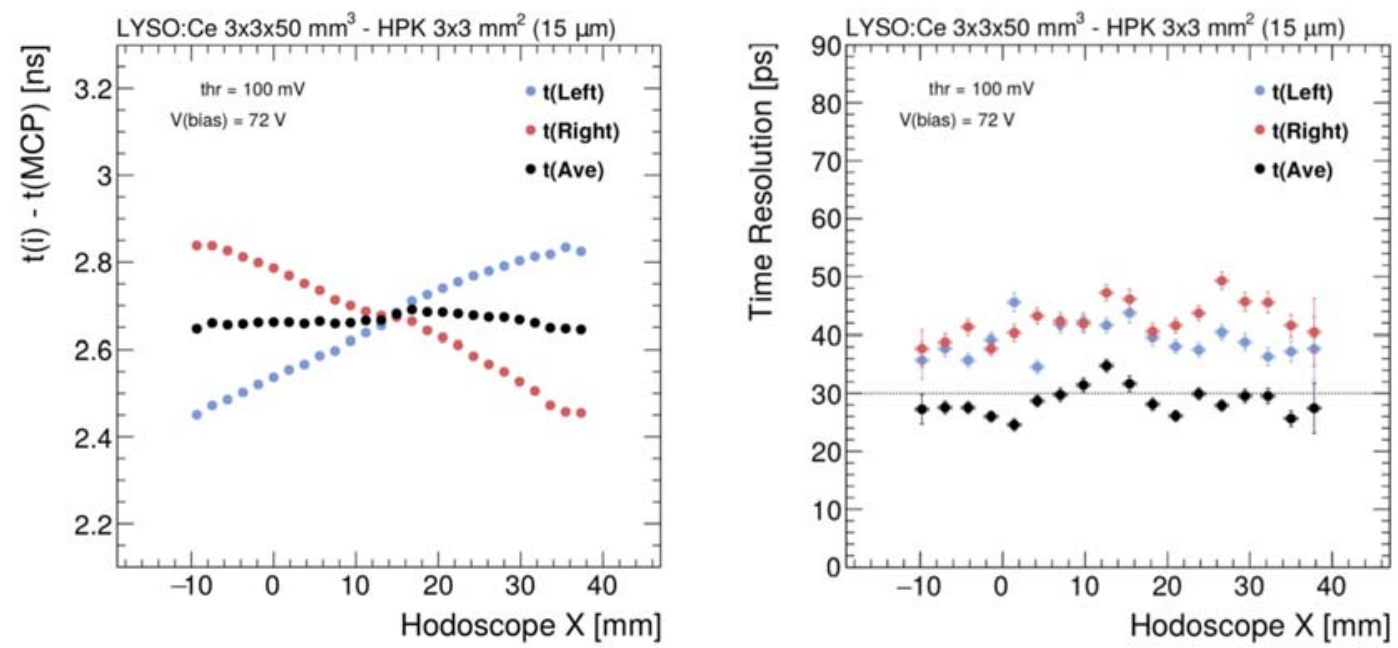

Figure 2 Timing resolution of CMS LYSO Scintillator rod

unit was originally developed for ToF-PET (Positron Emission Tomography) measurement with $\mathrm{PPD} / \mathrm{SiPM}$ [5], where the two photon vertex is reconstructed better using time of flight (ToF) information of photons. PPD/SiPM is an acronym for pixelated photon detector (PPD) or Silicon Photon Multiplier (SiPM), in which an array of avalanche photo diode pixels are operated in a Geiger mode. [6] [7] In the course of the development, performance of PPD/SiPM has been improved very much in many respects, including timing information, as shown in Fig. 3. [8]
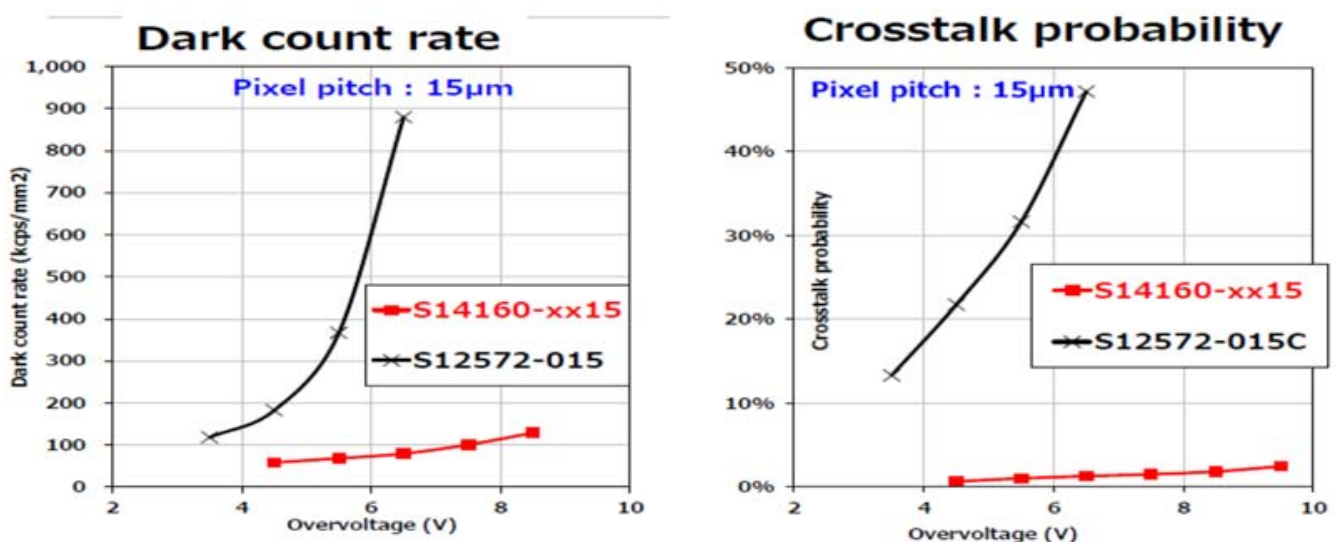

Figure 3 Improvements of PPD/SiPM in terms of dark count rate (left) or cross talk probability (right) as a function of over voltage (voltage exceeding Geiger breaking). The red line represents the 1st generation device while the black line represents the 3rd (current) generation. 
In order to test timing detectors in a test beam, a better timing reference detector with ultimate resolution is necessary. Such a resolution has been achieved so far by combining a Cherenkov detector with a MCP (Multi Channel Plate) photo mutiplier tube for photon detection [9]. The recent report by L. Sohl [10] gives a 4 picosecond resolution in a rather restricted area of $5 \mathrm{~mm}$.

It should be noted that such excellent timing measurement is demanded not only in HEP experiments but also in many near-future daily-life applications. One good example is LiDAR (Light Detection And Ranging) technology, which is an essential tool for automotive systems to reliably detect objects within a range of $\sim 100$ meters. LiDAR is based on Time of Flight (ToF) measurement of very faint light, which is familiar to an HEP experimentalist. Timing precision of some tens of picoseconds is necessary for a ranging resolution of $\mathrm{cm}$. LiDAR will also be a very powerful tool for facial recognition beyond 2D imaging. By adding depth information, accuracy and reliability of recognition can be dramatically enhanced. A practical application in depth measurement using ToF requires a timing resolution better than 10 picoseconds in every pixel. There is an impressive report for an industrial ranging application [11] describing that a prototype array of $132 \times 120$ photo diode pixels sucessfully demonstrates a timing resolution of 1.7 picoseconds, equivalent to $0.25 \mathrm{~mm}$ resolution in depth measurement, as shown in Fig. 4, where an independent timing measurement is done in each pixel electronics making triple captures of a pulse shape.

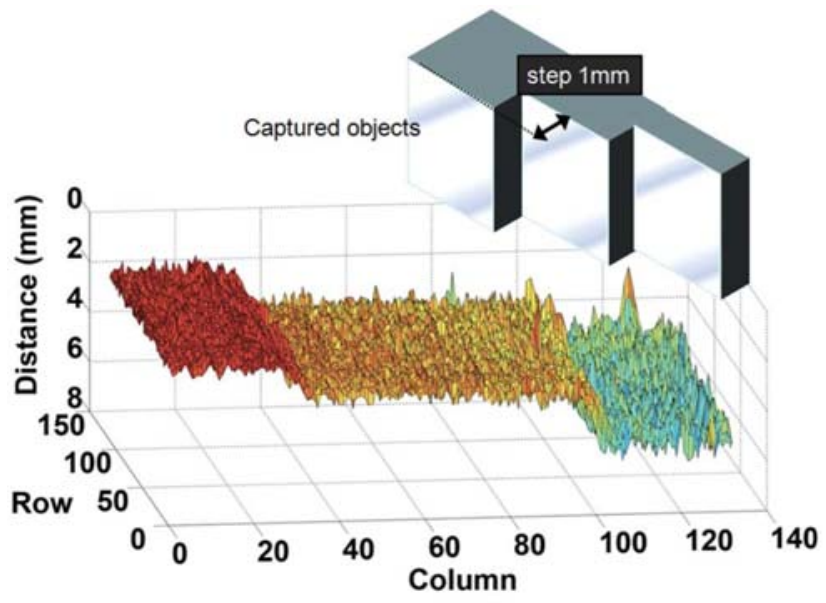

Figure 4 Time of Flight imaging with a pixel array sensor for depth measurement.

\section{Space measurement}

Better tracking with higher spatial resolution is always crucial for event reconstruction/pile up mitigation under higher particle flux, better momentum measurement or better heavy flavor tagging with vertex reconstruction. Although there has been remarkable progress in the field of gaseous detectors among vast varieties of tracking detectors, it must be skipped in this short review because the saga is too tremendous to be told fully.

One impressive recent progress is a scintillation fiber tracker system developed by the LHCb group. [12] The tracker plane is composed of a mat of six layers of $250 \mu \mathrm{m}$

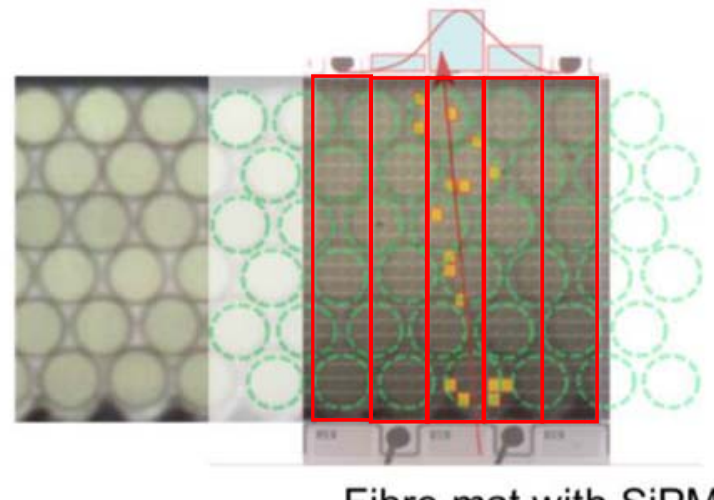

Fibre mat with SiPM

Figure 5 LHCb SciFi tracker cross section with PPD/SiPM photon sensors (illustrated by red boxes) overlayed. 
$\phi$ scintillation fibers (SciFi) and specially designed PPD (SiPM) aligning each narrow rectangular array of $4 \times 26$ pixels to cover $250 \mu \mathrm{m}$ (wide) x $1.3 \mathrm{~mm}$ (high) area in the end plane of the SciFi mat, as shown in Fig. 5.

Thanks to multiple measurements penetrating six layers of SciFi, the expected number of photons in a mat is 16 20 for a single particle incidence even at the far end ( $2.4 \mathrm{~m}$ from sensors), which is sufficient to locate the track with very high efficiency. The track localization resolution has been examined by the beam test to be $\sim 70 \mu \mathrm{m}$, effectively consistent with a binary readout of strips with pitch of $250 \mu \mathrm{m}$, as shown in Fig. 6. [13]

The vertex detector is the field where ultimate spatial resolution is required. To accommodate very high particle flux at the location of vertex detectors, pixel sensors are used in the current LHC experiments at the innermost region. Those pixel sensor system are, however, rather thick hybrid devices, in which sensor diode chips and readout electronics chips are bump bonded. The bump bonding technology may introduce thicker

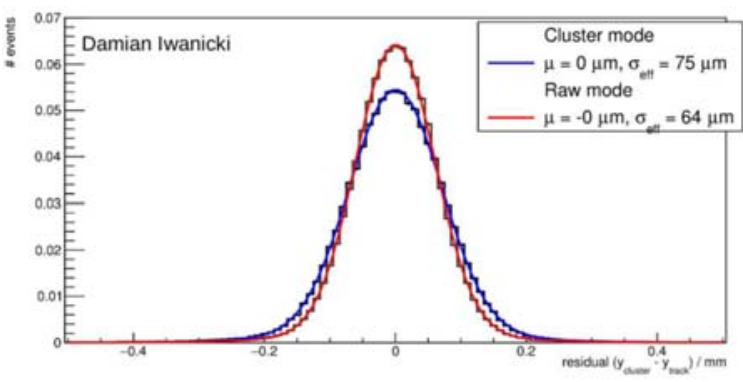

Figure 6 Single hit resolution of LHCb SciFi tracker. material in a sensitive area and restrict smaller pixel pitches to maintain reasonable bonding yields. Another serious concern is a rather complicated assembly procedure of hybrid pixel detectors due to bonding. To avoid all those difficulties, a monolithic pixel device has been considered to be a "next generation" pixel sensor. It is interesting to know that the best impact parameter resolution in the history was achieved in the 1990s by the SLD vertex detector system composed of CCDs [14], the very first monolithic pixel sensor. To set a new record in the 21 century, a new type of thin monolithic pixel system of better spatial resolution with smaller pixel size should be realized. There are three technologies of modern monolithic pixel sensor in development, namely, CMOS MAPS [15] [16], DEPFET [17] [18] and SOI (Silicon-on-Insulator) pixel [19] [20]. The first two have a longer history and some practical applications in real experiments. CMOS MAPS has already demonstrated good performance in the STAR experiment in RHIC. The newly installed Belle II vertex detector system (VXD) consisting of two layers of DEPFET pixel sensor and four layers of silicon strip sensor is expected to surpass the great SLD VXD record after 25 years.

An ultimate spatial resolution in a pixel sensor could be realized by a centroid of proper charge sharing among fine-pitch pixels according to the formula, $(\Delta X)^{2}=\left(\frac{\text { pixel pitch }}{\text { SNR }}\right)^{2}$. In this respect, low-noise charge sharing among deeply depleted sensing nodes is essential for the best spatial resolution. The best resolution so far reported was that achieved by the SOI pixel sensor with pixel pitch of $8 \mu \mathrm{m}$, very deep depletion layer of $\sim 400 \mu \mathrm{m}$, operating at rather high SNR (signal to noise ratio) of 343 [21]. Fig. 7 indicates that its intrinsic resolution has reached true sub-micron $(\sim 0.6 \mu \mathrm{m})$ level for charged particle tracking.

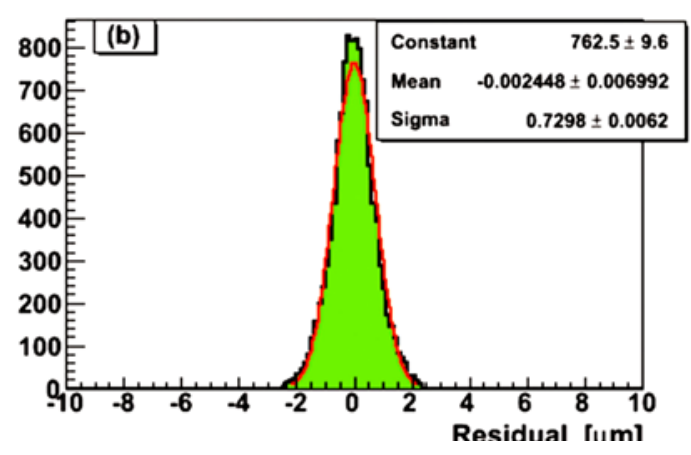

Figure 7 Track residual distribution of the SOI pixel beam test. 
Timing information is another important key issue for pixel sensors in the next generation because an effective reduction of hit occupancy using narrower time windows should be essential at higher luminosity colliders. The current popular monilithic pixel technologies like CMOS MAPS nor DEPFET have not yet provided satisfactory solutions in this respect since they are not working in a "pulse mode" but in an asynchronous "rolling shutter" mode. For good timing information with faster device response, a quick collection of signal charge in a deeper depletion layer will be very useful. Several new types of CMOS-MAPS with a special well structure enabling deeper depletion or an SOI pixel sensor intrinsically having depletion of any depth are considered as a promising candidate. [22]

\section{Radiation tolerance}

Tolerance against high radiation environments is a critical requirement for particle detectors to be used in higher luminosity colliders. As known well there are two important phenomena degrading performance of detectors: Total Ionization Dose (TID) and Non Ionizing Energy Loss (NIEL). The former is related to the accumulation of ionized charge at some interface in the structure of irradiated device which might cause an erroneous local field and disturb normal operation. The latter is caused by a nucleon or a heavier ion which might hit atoms of detector material violently off their nomal position in the lattice and leave some defect, which may deteriorate their performance as a detector. The damage caused by those phenomena is given as a function of integration of accumulated radiation dose. It is generally considered that TID is more serious in a lepton collider while NIEL in a hadron collider.

Another important effect of harsh radiation is so called Single Event Effect (SEE). This is a phenomena in which single incoming radiation causes a big incident effect like very heavy ionization loss or a spallation of nucleus and affects critical nodes of devices to mulfunction (bit inversion, false trigger and so on). Even in the current operation of ATLAS or CMS, the effects are very serious, as shown in Fig. 8

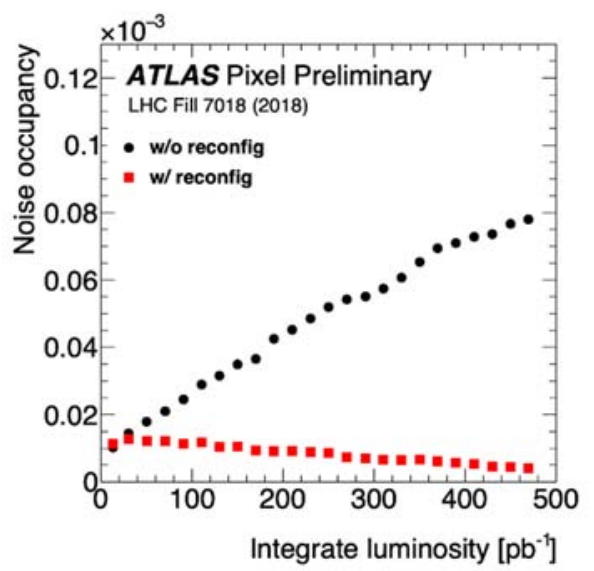

Figure 8 Growth of noise occupancy as a function of integrated luminosity (time) with and without continuous (every $5 \mathrm{sec}$ ) reconfiguration of registers. where very rapid growth of false hits is observed due to bit inversions happening in some registers of ATLAS pixel electronics under very high radiation. A frequent (every $5 \mathrm{sec}$ ) reconfiguration of the registers can recover the effect dramatically, as shown in red points in the figure. [23] 


\section{Detectors for neutrino}

The modern neutrino detector systems like Super- or Hyper-Kamiokande [24], IceCube [25], KM3Net [26] or JUNO [27] are all composed of state-ofthe-art but legendary vacuum tubes (photo multiplier tube, PMT) as a photon sensor. It is very interesting to see still big improvements are steadily made for PMT in terms of detection efficiency, noise and timing characteristics. Fig. 8 shows such improvements made in the SuperKamiokande PMT for the new PMT

Total Detection Efficiency of 1pe

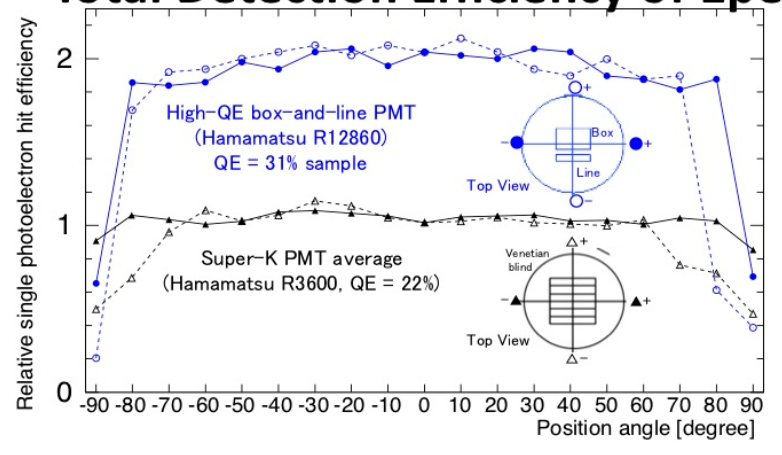

Figure 9 Detection efficiency improvement from SuperKamiokande to HyperKamiokande PMT. to be used in HyperKamiokande. Twice higher detection efficiency has been obtained by a new dynode structure and a new photocathode technology [24]. Those big improvements would help to boost the performance of the detector system for a better measurement or to dramatically reduce the cost of the detetor system keeping the performance as initially expected.

\section{Conclusion}

The space-time performance of particle detectors is steadily advancing and now coming into the era of precisions of "sub pico-second" and "sub micro-meter". It is, however, a fair observation that the particle detector is no longer a key player to drive developments of cuttingedge technologies for society as before, even though the research and development in particle physics experiments could still provide the most critical and scientific characterization and evaluation for the technology development.

Particle detectors are now working in two extreme environments, very harsh radiation at human-made accelerators with highest precision or very low background activity at deep underground labs with highest sensitivity. Many of the advanced technologies common to both areas are developed actively. Sustainable R\&D of detectors and instrumentation should be carried out as a horizontal activity among an entire particle physics community.

The author would like to express his thanks to Drs. Kazunori Hanagaki (KEK), Daniel Jean (KEK), Masashi Yokoyama (Tokyo), Atsuhiko Ochi (Kobe) and Kentaro Miuchi(Kobe) for providing useful materials and kind suggestions in preparing this contribution.

\section{References}

[1] N. Wermes, CMOS sensor development in Bonn, Belle II VXD Open Workshop, 503/1-001 - Council Chamber (CERN), 8 Jul 2019 - 10 Jul 2019.

[2] CMS Collaboration, Initial Report of the Fast Timing Working Group, CERN-CMS-DP-2016-008, 
2016.

[3] H.F.W. Sadrozinski, A. Seiden and N. Cartiglia, 4D tracking with ultra-fast silicon detectors, Rep. Progr. Phys., 81 (2018).

[4] M. T. Lucchini, Development of the CMS Mip Timing Detector, VCI2019 - The 15th Vienna Conference on Instrumentation, Vienna, 2019.

[5] C. Piemonte, SiPM technology at FBK, 12th Topical Seminar on Innovative Particle and Radiation Detectors , Siena, 2010.

[6] J. Haba, Status and perspectives of Pixelated Photon Detector (PPD), Nuclear Instruments and Methods in Physics Research Section A:, 595 (2008) pp. 154-160.

M. Tanabashi et al. (Particle Data Group), The Review of Particle Physics (2019), Phys. Rev. D 98 (2018) 030001.

[7] F. Acerbi, SiPM overview: status and trends, 5th International Workshop on New Photon-Detectors (PD18), Tokyo, 2018.

[8] K. Yamamoto, Recent development of MPPC and Si detectors for HEP experiments, 5th International Workshop on New Photon-Detectors (PD18), Tokyo, 2018.

[9] K. Inami, N. Kishimoto, Y. Enari, M. Nagamine and T.Ohshima, A 5 ps TOF-counter with an MCP$P M T$, Nuclear Instruments and Methods in Physics Research Section A:, 560, (2006) 303.

[10] L. Sohl, Spatial time resolution of MCP-PMTs as a t0-reference, Nuclear Instruments and Methods in Physics Research Section A:, 936, (2019) 583.

[11] Keita Yasutomi, Takahiro Usui, Sang-Man Han, Taishi Takasawa, Keiichiro Kagawa and Shoji Kawahito, A Submillimeter Range Resolution Time-of-Flight Range Imager With Column-Wise Skew Calibration, IEEE Transactions on Electron Devices, 63 (2016) 182 - 188.

[12] L. Gruber, LHCb SciFi - Upgrading LHCb with a scintillating fibre tracker, Nuclear Instruments and Methods in Physics Research Section A:, In press, 2019.

[13] A. Comerma, PACIFIC: The readout ASIC for the SciFi Tracker, TWEPP, 2018.

[14] N. B. Sinev et al., Initial impact parameter resolution of the new SLD vertex detector, Nuclear Instruments and Methods in Physics Research Section A:, 409 (1998) 243.

[15] R. Turchetta et al., A monolithic active pixel sensor for charged particle tracking and imaging using standard VLSI CMOS technology, Nuclear Instruments and Methods in Physics Research Section A:, 458 (2001) 677.

[16] A. Besson et al., From vertex detectors to inner trackers with CMOS pixel sensors, Nuclear Instruments and Methods in Physics Research Section A:, 845 (2017) 33-37.

[17] J. Kemmer and G. Lutz New detector concepts, Nuclear Instruments and Methods in Physics Research Section A:, 253 (1987) 365.

[18] F. Abudinen et al., DEPFET pixel detector in the Belle II experiment, Nuclear Instruments and Methods in Physics Research Section A:, 936 (2019) 657.

[19] Y. Arai et al., Development of SOI pixel process technology, Nuclear Instruments and Methods in Physics Research Section A:, 636 (2011) 531.

[20] I. Kurachi and Y. Arai, Radiation Imaging Detectors Using Soi Technology, Morgan \& Claypool, 2017.

[21] D. Sekigawa et al., Fine-Pixel Detector FPIX Realizing Sub-micron Spatial Resolution Developed Based on FD-SOI Technology, in proceedings of International Conference on Technology and Instrumentation in Particle Physics 2017, 2 (2017) 331.

[22] W. Snoeys, Development of monolithic sensors for high energy physics in commercial CMOS technologies, Nuclear Instruments and Methods in Physics Research Section A:, 938 (2019) 41-50.

[23] ATLAS Collaboration, Public Pixel Tracker Plots for Collision Data, PIX-2018-010," 2018. Available: https://atlas.web.cern.ch/Atlas/GROUPS/PHYSICS/PLOTS/PIX-2018-010/.

[24] K. Abe et al., Hyper-Kamiokande Proto-Collaboration, HyperKamiokande Design Report, arXiv:1805.04163v2 [physics.ins-det], 2018.

[25] The IceCube Collaboration, M. G. Aartsen et al., Measurement of the multi-TeV neutrino interaction 
cross-section with IceCube using Earth absorption, Nature, 551 (2017)596-600.

[26] M. de Jong, KM3NeT 2.0: A carbon neutral research facility, Science and Technology of Pan European Networks, 22 (2017) 108-109.

[27] Yu-Feng Li, Jun Cao, Yifang Wang and Liang Zhan, Unambiguous determination of the neutrino mass hierarchy using reactor neutrinos, Phys. Rev. D, 88 (2013) 013008. 